\title{
An expedient total synthesis of (-)-dactylolide and formal synthesis of (-)-zampanolide
}

\section{Fei Ding and Michael P. Jennings*}

Department of Chemistry, University of Alabama, 500 Campus Dr.

Tuscaloosa, AL 35487-0336

jenningm@bama.ua.edu

\section{Supporting Information}

General Procedure ${ }^{1} \mathrm{H}$ and ${ }^{13} \mathrm{C}$ NMR spectra were collected on a $360 \mathrm{MHz}$ or 500 $\mathrm{MHz}$ Bruker spectrometer. Chemical shifts were reported in parts per million relative to tetramethylsilane (TMS) using deuterated chloroform $\left(\mathrm{CDCl}_{3}\right)$ as the internal reference, and coupling constants were reported in Hertz. Optical rotation measurements were performed on a Rudolph AUTOPOL IV polarimeter at Biocryst Pharmaceuticals, Inc. FTIR spectra were recorded on a BIO-RAD FTS-40 spectrometer. HRMS determination was performed in the laboratory of mass spectrometry at the University of Illinois, Urbana-Champaign. Solvents and reagents were used as purchased without further purification.

5,6-Bis-(tert-butyl-dimethyl-silanyloxy)-hex-2-ynoic acid ethyl ester (6) $\quad$ To a flame-dried flask under Ar, a BuLi solution (1.6 M in hexanes, $21 \mathrm{~mL}, 33.7 \mathrm{mmol}$ ) was added dropwise into a solution of the alkyne $(7.37 \mathrm{~g}, 22.5 \mathrm{mmol})$ in THF $(63 \mathrm{~mL})$ at $78^{\circ} \mathrm{C}$ and stirred for $15 \mathrm{~min}$. Ethyl chloroformate $(6.45 \mathrm{~mL}, 67.5 \mathrm{mmol})$ was then added, and the solution was warmed to rt and kept stirring for $45 \mathrm{~min}$ before quenched with a 
saturated $\mathrm{NaHCO}_{3}$ solution $(60 \mathrm{~mL})$. The aqueous solution was extracted with $\mathrm{Et}_{2} \mathrm{O}(3 \mathrm{X}$ $60 \mathrm{~mL}$ ). The combined organic solution was dried over $\mathrm{Na}_{2} \mathrm{SO}_{4}$ and concentrated under reduced pressure. Flash chromatography (silica, 2\% EtOAc in hexanes) afforded the alkynoate $\left(\mathrm{R}_{\mathrm{f}}=0.40,2 \%\right.$ EtOAc in hexanes) as colorless oil (9.5 g, 100\%). ${ }^{1} \mathrm{H}$ NMR (360 $\left.\mathrm{MHz}, \mathrm{CDCl}_{3}\right) \delta 4.21(\mathrm{q}, \mathrm{J}=7.2,2 \mathrm{H}), 3.87(\mathrm{~m}, 1 \mathrm{H}), 3.59(\mathrm{dd}, \mathrm{J}=10.2,4.9,1 \mathrm{H}), 3.48(\mathrm{dd}$, $\mathrm{J}=10.2,6.9,1 \mathrm{H}), 2.65(\mathrm{dd}, \mathrm{J}=17.0,4.7,1 \mathrm{H}), 2.43(\mathrm{dd}, \mathrm{J}=17.0,6.6,1 \mathrm{H}), 1.29(\mathrm{t}, \mathrm{J}=$ 7.2, 3H), 0.89 (s, 18H), 0.12 (s, 3H), 0.08 (s, 3H), 0.06 (s, 6H). ${ }^{13} \mathrm{C}$ NMR (90 MHz, $\left.\mathrm{CDCl}_{3}\right) \delta 154.9,87.2,74.8,71.4,66.7,62.1,35.2,26.1,25.0,23.3,18.3,14.2,-4.3,-4.6,-$ 5.1, -5.2. $[\alpha]^{20}{ }_{D}-6.4\left(\mathrm{c} 0.90, \mathrm{CH}_{2} \mathrm{Cl}_{2}\right)$. IR $\left(\mathrm{CH}_{2} \mathrm{Cl}_{2}\right): 1710,1471,1115,1082 \mathrm{~cm}^{-1}$. HRMS (ESI) calcd for $\mathrm{C}_{20} \mathrm{H}_{41} \mathrm{O}_{4} \mathrm{Si}_{2}(\mathrm{M}+\mathrm{H})^{+}$: 401.2543, found: 401.2528 .

\section{5,6-Bis-(tert-butyl-dimethyl-silanyloxy)-3-methyl-hex-2-enoic acid ethyl ester (7)}

To a solution of $\mathrm{NaOMe}(61 \mathrm{mg}, 1.1 \mathrm{mmol})$ in $\mathrm{MeOH}(54 \mathrm{~mL})$, were added $\mathrm{PhSH}$ (2.86 $\mathrm{mL}, 28.0 \mathrm{mmol})$ and the alkynoate $(9.0 \mathrm{~g}, 22.5 \mathrm{mmol})$. The solution was kept stirring for $20 \mathrm{~h}$ at rt, before filtered through a silica plug, which was then eluted with $\mathrm{Et}_{2} \mathrm{O}$ to bring down the yellow band. Evaporation of solvents yielded the crude product. Flash chromatography (silica, $10 \%$ EtOAc in hexanes) afforded the alkenoate $\left(\mathrm{R}_{\mathrm{f}}=0.40,10 \%\right.$ EtOAc in hexanes) as pale yellow oil (10.35 g, 90\%). ${ }^{1} \mathrm{H} \mathrm{NMR}\left(360 \mathrm{MHz}, \mathrm{CDCl}_{3}\right) \delta 7.51$ (m, 2H), $7.35(\mathrm{~m}, 3 \mathrm{H}), 5.94(\mathrm{~s}, 1 \mathrm{H}), 4.21(\mathrm{~m}, 2 \mathrm{H}), 3.63(\mathrm{~m}, 1 \mathrm{H}), 3.33(\mathrm{dd}, \mathrm{J}=10.0,5.0$, $1 \mathrm{H}), 3.15(\mathrm{dd}, \mathrm{J}=10.0,5.6,1 \mathrm{H}), 1.29(\mathrm{t}, \mathrm{J}=7.2,3 \mathrm{H}), 0.82(\mathrm{~s}, 9 \mathrm{H}), 0.79(\mathrm{~s}, 9 \mathrm{H}),-0.01(\mathrm{~s}$,

3H), -0.04 (s, 3H), -0.09 (s, 6H). ${ }^{13} \mathrm{C} \mathrm{NMR}\left(90 \mathrm{MHz}, \mathrm{CDCl}_{3}\right) \delta 166.1,156.9,135.6$, $131.5,129.4,115.9,71.8,67.6,60.1,41.8,18.3,15.5,14.3,-4.2,-4.6,-5.2,-5.3$.

To a flame-dried flask under $\mathrm{Ar}$, a $\mathrm{MeMgBr}$ solution (3.0 $\mathrm{M}$ in $\mathrm{Et}_{2} \mathrm{O}, 473 \mu \mathrm{L}$, $1.42 \mathrm{mmol})$ was added dropwise into a suspension of CuI (296 mg, $1.56 \mathrm{mmol})$ in THF 
(4.7 $\mathrm{mL}$ ) at $-78^{\circ} \mathrm{C}$. The reaction mixture was warmed to $\mathrm{rt}$ and then cooled back to $-78^{\circ} \mathrm{C}$, before a solution of the thioether $(0.50 \mathrm{~g}, 0.98 \mathrm{mmol})$ in THF $(0.5 \mathrm{~mL})$ was added. The solution was warmed to $0^{\circ} \mathrm{C}$ and kept stirring for $1 \mathrm{~h}$. Water $(0.5 \mathrm{~mL})$ was then added, and the mixture was diluted with $\mathrm{Et}_{2} \mathrm{O}(20 \mathrm{~mL})$, dried over $\mathrm{Na}_{2} \mathrm{SO}_{4}$. The solution was filtered through a silica plug, which was then eluted with $\mathrm{Et}_{2} \mathrm{O}(10 \mathrm{~mL})$. The combined organics were concentrated under reduced pressure to afford the product $\left(\mathrm{R}_{\mathrm{f}}=0.55,10 \%\right.$

EtOAc in hexanes) as colorless oil without further purification (395 mg, 97\%). ${ }^{1} \mathrm{H}$ NMR $\left(360 \mathrm{MHz}, \mathrm{CDCl}_{3}\right) \delta 5.70(\mathrm{~s}, 1 \mathrm{H}), 4.14(\mathrm{~m}, 2 \mathrm{H}), 3.81(\mathrm{~m}, 1 \mathrm{H}), 3.54(\mathrm{dd}, \mathrm{J}=9.9,5.0,1 \mathrm{H})$, $3.37(\mathrm{dd}, \mathrm{J}=9.9,6.8,1 \mathrm{H}), 2.45(\mathrm{dd}, \mathrm{J}=12.7,3.8,1 \mathrm{H}), 2.18(\mathrm{~d}, \mathrm{~J}=1.4,3 \mathrm{H}), 2.15(\mathrm{dd}, \mathrm{J}=$ 12.7, 7.9, 1H), $1.26(\mathrm{t}, \mathrm{J}=7.0,3 \mathrm{H}), 0.89(\mathrm{~s}, 9 \mathrm{H}), 0.86(\mathrm{~s}, 9 \mathrm{H}), 0.05(\mathrm{~s}, 6 \mathrm{H}), 0.03(\mathrm{~s}, 3 \mathrm{H})$, 0.0 (s, 3H). ${ }^{13} \mathrm{C}$ NMR $\left(90 \mathrm{MHz}, \mathrm{CDCl}_{3}\right) \delta 166.8,156.9,118.5,71.8,67.3,59.6,46.2$, $26.2,26.0,25.7,19.7,18.5,18.3,15.5,14.3,-4.2,-4.7,-5.1,-5.2 .[\alpha]^{20}{ }_{D}-7.7$ (c 2.35, $\left.\mathrm{CH}_{2} \mathrm{Cl}_{2}\right)$. IR $\left(\mathrm{CH}_{2} \mathrm{Cl}_{2}\right): 1713,1653,1474,1226,1154,1094 \mathrm{~cm}^{-1}$. HRMS (ESI) calcd for $\mathrm{C}_{21} \mathrm{H}_{45} \mathrm{O}_{4} \mathrm{Si}_{2}(\mathrm{M}+\mathrm{H})^{+}: 417.2856$, found: 417.2860.

5,6-Bis-(tert-butyl-dimethyl-silanyloxy)-3-methyl-hex-2-enal (8) To a flamedried flask under Ar, a DIBAL-H solution (1.0 M in $\mathrm{CH}_{2} \mathrm{Cl}_{2}, 2.2 \mathrm{~mL}, 2.2 \mathrm{mmol}$ ) was added dropwise into a solution of the ester $(416 \mathrm{mg}, 1.0 \mathrm{mmol})$ in $\mathrm{CH}_{2} \mathrm{Cl}_{2}(7.8 \mathrm{~mL})$ at $78^{\circ} \mathrm{C}$ and stirred for $2 \mathrm{~h}$. The solution was then poured directly into a stirring mixture of a saturated sodium potassium tartrate aqueous solution $(3 \mathrm{ml})$ and hexanes $(17 \mathrm{~mL})$. The aqueous layer was extracted with $\mathrm{Et}_{2} \mathrm{O}(10 \mathrm{~mL})$, and the combined organics were filtered through a celite plug. Evaporation of solvents afforded the alcohol $\left(R_{f}=0.20,20 \%\right.$ EtOAc in hexanes) as colorless oil without further purification $(354 \mathrm{mg}, 95 \%) .{ }^{1} \mathrm{H} \mathrm{NMR}$ $\left(360 \mathrm{MHz}, \mathrm{CDCl}_{3}\right) \delta 5.45(\mathrm{t}, \mathrm{J}=7.1,1 \mathrm{H}), 4.15(\mathrm{~m}, 2 \mathrm{H}), 3.77(\mathrm{~m}, 1 \mathrm{H}), 3.51(\mathrm{dd}, \mathrm{J}=9.9$, 
5.4, 1H), $3.39(\mathrm{dd}, \mathrm{J}=9.9,6.3,1 \mathrm{H}), 2.31(\mathrm{dd}, \mathrm{J}=13.3,4.2,1 \mathrm{H}), 2.06(\mathrm{dd}, \mathrm{J}=13.3,7.5$, 1H), 1.70 (s, 3H), 0.89 (s, 9H), 0.86 (s, 9H), 0.04 (s, 9H), 0.02 (s, 3H). ${ }^{13} \mathrm{C}$ NMR (90 $\left.\mathrm{MHz}, \mathrm{CDCl}_{3}\right) \delta 136.8,126.6,72.1,67.5,59.7,44.9,26.2,26.1,25.9,23.6,18.6,17.2,-$ 4.1, -4.6, -5.06, -5.13. $[\alpha]^{20}{ }_{D}-4.4\left(\mathrm{c} 0.90, \mathrm{CH}_{2} \mathrm{Cl}_{2}\right) . \mathrm{IR}\left(\mathrm{CH}_{2} \mathrm{Cl}_{2}\right): 1473,1226,1154,1094$ $\mathrm{cm}^{-1}$. HRMS (ESI) calcd for $\mathrm{C}_{19} \mathrm{H}_{43} \mathrm{O}_{3} \mathrm{Si}_{2}(\mathrm{M}+\mathrm{H})^{+}: 375.2751$, found: 375.2756 .

To a solution of the allylic alcohol $(151 \mathrm{mg}, 0.404 \mathrm{mmol})$ and $\mathrm{NaOAc}(27 \mathrm{mg}$, $0.324 \mathrm{mmol})$ in $\mathrm{CH}_{2} \mathrm{Cl}_{2}(2 \mathrm{~mL})$, was added $\mathrm{PCC}(174 \mathrm{mg}, 0.808 \mathrm{mmol})$. The reaction mixture was stirred for $1 \mathrm{~h}$ at $\mathrm{rt}$, before diluted with $\mathrm{Et}_{2} \mathrm{O}(10 \mathrm{~mL})$ and then filtered through a silica plug. The plug was washed with $\mathrm{Et}_{2} \mathrm{O}(4 \mathrm{~mL})$. Evaporation of solvents afforded the enal $\left(\mathrm{R}_{\mathrm{f}}=0.50,20 \%\right.$ EtOAc in hexanes) as yellowish oil $(141 \mathrm{mg}, 94 \%, 6: 1$ Z:E). ${ }^{1} \mathrm{H}$ NMR (360 MHz, $\left.\mathrm{CDCl}_{3}\right) \delta 9.99(\mathrm{~d}, \mathrm{~J}=8.0,1 \mathrm{H}), 5.92(\mathrm{~d}, \mathrm{~J}=8.0,1 \mathrm{H}), 3.86(\mathrm{~m}$, $1 \mathrm{H}), 3.56(\mathrm{dd}, \mathrm{J}=9.8,4.7,1 \mathrm{H}), 3.37(\mathrm{dd}, \mathrm{J}=9.8,7.2,1 \mathrm{H}), 2.53(\mathrm{dd}, \mathrm{J}=13.3,4.5,1 \mathrm{H})$, $2.21(\mathrm{~s}, 3 \mathrm{H}), 0.89$ (s, 9H), $0.86(\mathrm{~s}, 9 \mathrm{H}), 0.051(\mathrm{~s}, 6 \mathrm{H}), 0.045(\mathrm{~s}, 3 \mathrm{H}), 0.01(\mathrm{~s}, 3 \mathrm{H}) .{ }^{13} \mathrm{C}$ NMR (90 MHz, $\left.\mathrm{CDCl}_{3}\right) \delta 191.4,161.6,130.2,72.0,67.1,46.0,26.1,14.3,-4.2,-4.6,-$ 5.13, -5.15. $[\alpha]^{20}{ }_{D}-2.4\left(\mathrm{c} 1.83, \mathrm{CH}_{2} \mathrm{Cl}_{2}\right)$. IR $\left(\mathrm{CH}_{2} \mathrm{Cl}_{2}\right): 1672,1469,1108 \mathrm{~cm}^{-1}$. HRMS (ESI) calcd for $\mathrm{C}_{19} \mathrm{H}_{41} \mathrm{O}_{3} \mathrm{Si}_{2}(\mathrm{M}+\mathrm{H})^{+}: 373.2594$, found: 373.2589 .

\section{8,9-Bis-(tert-butyl-dimethylsilanyloxy)-6-methyl-nona-1,5-dien-4-ol (9) To a} flame-dried flask under Ar, allylmagnesium bromide (1.0 M solution in ether, 29.0 mL) was added dropwise into a solution of (-)- $\mathrm{Ipc}_{2} \mathrm{BOMe}(10.0 \mathrm{~g}, 31.9 \mathrm{mmol})$ in anhydrous ether $(32 \mathrm{~mL})$ at $0^{\circ} \mathrm{C}$. The reaction mixture was stirred at $\mathrm{rt}$ for $1 \mathrm{~h}$ before cooled to $78^{\circ} \mathrm{C}$. The aldehyde $(7.18 \mathrm{~g}, 19.3 \mathrm{mmol})$ was added dropwise into the borane solution and allowed to stir for $1 \mathrm{~h}$ and then warm slowly to rt during $1 \mathrm{~h}$. An aqueous solution of $\mathrm{NaOH}\left(3 \mathrm{M}, 12.8 \mathrm{~mL}\right.$ ) was added, followed by slow addition of a $30 \% \mathrm{H}_{2} \mathrm{O}_{2}$ solution 
$(25.5 \mathrm{~mL})$. The mixture was refluxed for $3 \mathrm{~h}$. After cooled to rt, the biphasic solution was separated, and the aqueous layer was extracted with ether ( 3 X $50 \mathrm{~mL})$. The combined organics were dried over $\mathrm{Na}_{2} \mathrm{SO}_{4}$, and rotavapped to yield the crude product. Flash chromatography (silica, 10\% EtOAc in hexanes) afforded the homoallylic alcohol $\left(\mathrm{R}_{\mathrm{f}}=\right.$ $0.50,20 \%$ EtOAc in hexanes) as colorless oil (5.70 g, 71\%) along with other isomers (two diastereomers for each of the Z/E isomers, combined yield: $88 \%$, de: $90 \%$ ). ${ }^{1} \mathrm{H}$ NMR (360 MHz, $\left.\mathrm{CDCl}_{3}\right) \delta 5.81(\mathrm{~m}, 1 \mathrm{H}), 5.25(\mathrm{dd}, \mathrm{J}=8.5,1.0,1 \mathrm{H}), 5.12(\mathrm{~m}, 2 \mathrm{H}), 4.41$ $(\mathrm{dd}, \mathrm{J}=14.6,6.3,1 \mathrm{H}), 3.77(\mathrm{ddd}, \mathrm{J}=12.2,11.2,5.6,1 \mathrm{H}), 3.49(\mathrm{dd}, \mathrm{J}=10.0,4.5,1 \mathrm{H})$, $3.41(\mathrm{dd}, \mathrm{J}=10.0,4.4,1 \mathrm{H}), 2.31(\mathrm{dd}, \mathrm{J}=11.4,6.5,1 \mathrm{H}), 2.28(\mathrm{~m}, 2 \mathrm{H}), 2.05(\mathrm{dd}, \mathrm{J}=11.4$, 6.7, 1H), $1.72(\mathrm{~d}, \mathrm{~J}=1.2,3 \mathrm{H}), 0.90(\mathrm{~s}, 9 \mathrm{H}), 0.88(\mathrm{~s}, 9 \mathrm{H}), 0.055(\mathrm{~s}, 3 \mathrm{H}), 0.047(\mathrm{~s}, 6 \mathrm{H})$, $0.045(\mathrm{~s}, 3 \mathrm{H}) .{ }^{13} \mathrm{C} \mathrm{NMR}\left(90 \mathrm{MHz}, \mathrm{CDCl}_{3}\right) \delta 136.1,134.7,130.2,118.0,72.3,68.0,67.2$ $44.9,42.3,26.2,26.1,18.6,18.4,17.6,-4.05,-4.50,-5.05,-5.11 .[\alpha]^{20}{ }_{D}-10$ (c 1.3, $\left.\mathrm{CH}_{2} \mathrm{Cl}_{2}\right)$. IR $\left(\mathrm{CH}_{2} \mathrm{Cl}_{2}\right): 1471,1120,1092 \mathrm{~cm}^{-1}$. HRMS (ESI) calcd for $\mathrm{C}_{22} \mathrm{H}_{46} \mathrm{O}_{3} \mathrm{Si}_{2} \mathrm{Na}$ $(\mathrm{M}+\mathrm{Na})^{+}: 437.2883$, found: 437.2885 .

\section{6-[4,5-Bis-(tert-butyl-dimethyl-silanyloxy)-2-methyl-pent-1-enyl]-5,6-dihydro-}

pyran-2-one (11) To a flame-dried flask under Ar, acryloyl chloride $(2.23 \mathrm{~mL}, 27.6$ mmol) was added dropwise into a solution of the alcohol (5.70 g, $13.8 \mathrm{mmol}), \mathrm{Et}_{3} \mathrm{~N}$ (4.29 $\mathrm{mL}, 30.4 \mathrm{mmol})$, and DMAP ( $84 \mathrm{mg}, 0.70 \mathrm{mmol})$ in $\mathrm{CH}_{2} \mathrm{Cl}_{2}(138 \mathrm{~mL})$ at $\mathrm{rt}$. The solution was stirred for $16 \mathrm{~h}$, before brine $(100 \mathrm{~mL})$ was added. The aqueous layer was extracted with $\mathrm{CH}_{2} \mathrm{Cl}_{2}(2 \mathrm{X} 50 \mathrm{~mL})$, and the combined organics were washed with brine $(50 \mathrm{~mL})$ and then dried over $\mathrm{Na}_{2} \mathrm{SO}_{4}$. The solvents were removed under reduced pressure. Flash chromatography (silica, $2 \%$ EtOAc in hexanes) afforded the acrylate $\left(\mathrm{R}_{\mathrm{f}}=0.40,10 \%\right.$ EtOAc in hexanes) as colorless oil (5.12 g, 79\%). ${ }^{1} \mathrm{H}$ NMR $\left(360 \mathrm{MHz}, \mathrm{CDCl}_{3}\right) \delta 6.37$ (dd, 
$\mathrm{J}=17.2,1.3,1 \mathrm{H}), 6.07(\mathrm{dd}, \mathrm{J}=17.2,10.0,1 \mathrm{H}), 5.78(\mathrm{dd}, \mathrm{J}=10.0,1.3,1 \mathrm{H}), 5.68(\mathrm{~m}, 2 \mathrm{H})$, $5.24(\mathrm{dd}, \mathrm{J}=9.3,1.2,1 \mathrm{H}), 5.06(\mathrm{~m}, 2 \mathrm{H}), 3.75(\mathrm{ddd}, \mathrm{J}=12.5,11.6,5.6,1 \mathrm{H}), 3.48(\mathrm{dd}, \mathrm{J}=$ 10.0, 5.4, 1H), $3.38(\mathrm{dd}, \mathrm{J}=10.0,6.1,1 \mathrm{H}), 2.40(\mathrm{~m}, 2 \mathrm{H}), 2.31(\mathrm{dd}, \mathrm{J}=13.9,6.0,1 \mathrm{H})$, $2.06(\mathrm{dd}, \mathrm{J}=13.9,6.5,1 \mathrm{H}), 1.75(\mathrm{~d}, \mathrm{~J}=1.1,3 \mathrm{H}), 0.89(\mathrm{~s}, 9 \mathrm{H}), 0.85(\mathrm{~s}, 9 \mathrm{H}), 0.04(\mathrm{~s}, 6 \mathrm{H})$, $0.03(\mathrm{~s}, 3 \mathrm{H}), 0.0(\mathrm{~s}, 3 \mathrm{H}) .{ }^{13} \mathrm{C} \mathrm{NMR}\left(90 \mathrm{MHz}, \mathrm{CDCl}_{3}\right) \delta 165.7,138.3,133.7,130.8,129.5$, $125.7,118.2,71.8,70.7,67.3,45.0,39.6,26.2,26.1,18.6,18.3,17.7,-4.2,-4.6,-5,08,-$ 5.13. $[\alpha]^{20}{ }_{\mathrm{D}}-8.2\left(\mathrm{c} 0.17, \mathrm{CH}_{2} \mathrm{Cl}_{2}\right)$. IR $\left(\mathrm{CH}_{2} \mathrm{Cl}_{2}\right): 1715,1409,1201 \mathrm{~cm}^{-1}$. HRMS (ESI) calcd for $\mathrm{C}_{25} \mathrm{H}_{48} \mathrm{O}_{4} \mathrm{Si}_{2} \mathrm{Na}(\mathrm{M}+\mathrm{Na})^{+}$: 491.2989, found: 491.2995.

To a flame-dried flask under Ar, Grubbs' second generation catalyst (208 mg, $0.245 \mathrm{mmol}$ ) was added into a solution of the acrylate (2.29 g, $4.90 \mathrm{mmol})$ in $\mathrm{CH}_{2} \mathrm{Cl}_{2}$ (300 mL). The solution was heated under refluxing for $16 \mathrm{~h}$, and then the solvent was removed under reduced pressure. Flash chromatography (silica, 20\% EtOAc in hexanes) afforded the lactenone $\left(\mathrm{R}_{\mathrm{f}}=0.28,20 \%\right.$ EtOAc in hexanes) as colorless oil $(2.06 \mathrm{~g}, 96 \%)$. ${ }^{1} \mathrm{H}$ NMR $\left(360 \mathrm{MHz}, \mathrm{CDCl}_{3}\right) \delta 6.87(\mathrm{dddd}, \mathrm{J}=13.2,8.5,3.6,1.4,1 \mathrm{H}), 6.03(\mathrm{ddd}, \mathrm{J}=13.2$, $1.7,1.7,1 \mathrm{H}), 5.40(\mathrm{dd}, \mathrm{J}=8.4,0.9,1 \mathrm{H}), 5.15(\mathrm{~m}, 1 \mathrm{H}), 3.77(\mathrm{ddd}, \mathrm{J}=11.6,5.2,1.4,1 \mathrm{H})$, $3.51(\mathrm{dd}, \mathrm{J}=10.0,5.1,1 \mathrm{H}), 3.39(\mathrm{dd}, \mathrm{J}=10.0,6.5,1 \mathrm{H}), 2.37(\mathrm{~m}, 3 \mathrm{H}), 2.08(\mathrm{dd}, \mathrm{J}=13.5$, 7.0, 1H), $1.76(\mathrm{~d}, \mathrm{~J}=1.4,1 \mathrm{H}), 0.89(\mathrm{~s}, 9 \mathrm{H}), 0.88(\mathrm{~s}, 9 \mathrm{H}), 0.05(\mathrm{~s}, 12 \mathrm{H}) .{ }^{13} \mathrm{C}$ NMR $(90$ $\left.\mathrm{MHz}, \mathrm{CDCl}_{3}\right) \delta 164.6,144.9,140.6,124.9,122.0,75.0,72.4,67.1,44.6,29.9,26.2,26.1$, 18.5, 18.3, 18.1, -4.2, -4.5, -5.09, -5.12. $[\alpha]^{20}{ }_{\mathrm{D}}-12\left(\mathrm{c} 0.17, \mathrm{CH}_{2} \mathrm{Cl}_{2}\right) . \mathrm{IR}\left(\mathrm{CH}_{2} \mathrm{Cl}_{2}\right): 1719$, $1424 \mathrm{~cm}^{-1}$. HRMS (ESI) calcd for $\mathrm{C}_{23} \mathrm{H}_{45} \mathrm{O}_{4} \mathrm{Si}_{2}(\mathrm{M}+\mathrm{H})^{+}: 441.2856$, found: 441.2860 . 6-[4,5-Bis-(tert-butyl-dimethyl-silanyloxy)-2-methyl-pent-1-enyl]-4-hydroxytetrahydro-pyran-2-one (12) To a solution of the lactenone $(0.600 \mathrm{~g}, 1.36 \mathrm{mmol})$ in $\mathrm{MeOH}(6.8 \mathrm{~mL})$, was added an aqueous $\mathrm{H}_{2} \mathrm{O}_{2}$ solution $(30 \%, 488 \mathrm{mg}, 4.58 \mathrm{mmol})$. 
The solution was cooled to $0^{\circ} \mathrm{C}$, and an aqueous $\mathrm{NaOH}$ solution $(6.0 \mathrm{M}, 0.14 \mathrm{~mL}, 0.82$ mmol) was added dropwise and stirred for $10 \mathrm{~min}$. The reaction mixture was then warmed to rt and kept stirring for $0.5 \mathrm{~h}$, before diluted with $\mathrm{Et}_{2} \mathrm{O}(15 \mathrm{~mL})$ and $\mathrm{H}_{2} \mathrm{O}(15$ $\mathrm{mL}$ ). Concentrated aqueous $\mathrm{HCl}$ solution was added to adjust the $\mathrm{pH}$ to 4 . The aqueous layer was extracted with $\mathrm{Et}_{2} \mathrm{O}(2 \mathrm{X} 15 \mathrm{~mL})$, and the combined organics were washed with brine $(10 \mathrm{~mL})$. After dried over $\mathrm{Na}_{2} \mathrm{SO}_{4}$, the organic solution was concentrated under reduced pressure. The residue was re-dissolved in $\mathrm{PhH}(3.4 \mathrm{~mL})$, and the resultant solution was refluxed for 15 min using a Dean-Stark apparatus to remove water. After evaporation of $\mathrm{PhH}$, flash chromatography (silica, 20\% EtOAc in hexanes) afforded the epoxide $\left(R_{\mathrm{f}}=0.32,20 \%\right.$ EtOAc in hexanes $)$ as colorless viscous oil $(515 \mathrm{mg}, 83 \%) .{ }^{1} \mathrm{H}$ NMR (360 MHz, $\left.\mathrm{CDCl}_{3}\right) \delta 5.22(\mathrm{ddd}, \mathrm{J}=18.7,8.8,3.0,1 \mathrm{H}), 5.21(\mathrm{~d}, \mathrm{~J}=1.5,1 \mathrm{H}), 3.75$ $(\mathrm{ddd}, \mathrm{J}=11.8,5.0,1.8,1 \mathrm{H}), 3.66(\mathrm{dd}, \mathrm{J}=6.8,3.9,1 \mathrm{H}), 3.58(\mathrm{~d}, \mathrm{~J}=4.1,1 \mathrm{H}), 3.50(\mathrm{dd}, \mathrm{J}$ $=10.0,5.1,1 \mathrm{H}), 3.37(\mathrm{dd}, \mathrm{J}=10.0,6.6,1 \mathrm{H}), 2.33(\mathrm{~m}, 2 \mathrm{H}), 2.05(\mathrm{~m}, 2 \mathrm{H}), 1.73(\mathrm{~d}, \mathrm{~J}=0.7$, 3H), 0.89 (s, 9H), 0.87 (s, 9H), 0.044 (s, 3H), 0.040 (s, 6H), 0.035 (, 3H). ${ }^{13} \mathrm{C}$ NMR $(90$ $\left.\mathrm{MHz}, \mathrm{CDCl}_{3}\right) \delta 167.9,140.5,123.8,72.2,70.7,67.1,52.3,49.3,44.4,30.1,26.2,26.1$, 18.6, 18.3, 18.0, -4.2, -4.5, -5.08, -5.10, -5.12. $[\alpha]^{20}{ }_{D}+34\left(\mathrm{c} 0.37, \mathrm{CH}_{2} \mathrm{Cl}_{2}\right) . \mathrm{IR}\left(\mathrm{CH}_{2} \mathrm{Cl}_{2}\right)$ : $1738,1473,1363,1112,1080,1013 \mathrm{~cm}^{-1}$. HRMS (ESI) calcd for $\mathrm{C}_{23} \mathrm{H}_{45} \mathrm{O}_{5} \mathrm{Si}_{2}(\mathrm{M}+\mathrm{H})^{+}$: 457.2806, found: 457.2805 .

To a solution of $(\mathrm{PhSe})_{2}(482 \mathrm{mg}, 1.55 \mathrm{mmol})$ in $\mathrm{EtOH}(8.2 \mathrm{~mL})$ under Ar at rt, was added $\mathrm{NaBH}_{4}(118 \mathrm{mg}, 3.09 \mathrm{mmol})$ and stirred for $5 \mathrm{~min}$ before cooled to $0^{\circ} \mathrm{C}$. HOAc $(176 \mu \mathrm{L}, 3.09 \mathrm{mmol})$ was then added dropwise, and the solution was kept stirring for $5 \mathrm{~min}$ before a solution of the epoxide $(471 \mathrm{mg}, 1.03 \mathrm{mmol})$ in $\mathrm{EtOH}(6.2 \mathrm{~mL})$ was added. The reaction mixture was stirred for $15 \mathrm{~min}$, and then diluted with EtOAc $(36 \mathrm{~mL})$. 
The organic solution was washed with half-saturated $\mathrm{NaCl}$ solution $(9 \mathrm{~mL})$. The aqueous solution was then extracted with EtOAc $(2 \mathrm{X} 9 \mathrm{~mL})$ and the combined organic solution was dried over $\mathrm{Na}_{2} \mathrm{SO}_{4}$ and concentrated under reduced pressure. Flash chromatography (silica, 1:1 EtOAc:hexanes) afforded the alcohol $\left(\mathrm{R}_{\mathrm{f}}=0.30,1: 1\right.$ EtOAc:hexanes) as pale

yellow oil (368 mg, 78\%). ${ }^{1} \mathrm{H} \mathrm{NMR}\left(360 \mathrm{MHz}, \mathrm{CDCl}_{3}\right) \delta 5.42(\mathrm{ddd}, \mathrm{J}=8.8,8.8,3.4,1 \mathrm{H})$, $5.28(\mathrm{~d}, \mathrm{~J}=8.6,1 \mathrm{H}), 4.37(\mathrm{ddd}, \mathrm{J}=7.6,3.9,3.9,1 \mathrm{H}), 3.76(\mathrm{ddd}, \mathrm{J}=11.6,5.5,5.5,1 \mathrm{H})$, $3.50(\mathrm{dd}, \mathrm{J}=10.1,5.5,1 \mathrm{H}), 3.38(\mathrm{dd}, \mathrm{J}=10.1,6.4,1 \mathrm{H}), 2.71(\mathrm{dd}, \mathrm{J}=17.7,4.9,1 \mathrm{H}), 2.61$ (ddd, $\mathrm{J}=17.7,3.4,1.3,1 \mathrm{H}), 2.34(\mathrm{dd}, \mathrm{J}=13.4,4.9,1 \mathrm{H}), 2.07(\mathrm{dd}, \mathrm{J}=13.4,7.0,1 \mathrm{H})$, $1.93(\mathrm{ddd}, \mathrm{J}$ 14.2, 3.9, 3.9, 1H), $1.82(\mathrm{dd}, \mathrm{J}=14.2,3.4,1 \mathrm{H}), 1.76(\mathrm{~s}, 3 \mathrm{H}), 0.88$ (s, 9H), $0.86(\mathrm{~s}, 9 \mathrm{H}), 0.04(\mathrm{~s}, 12 \mathrm{H}) .{ }^{13} \mathrm{C} \mathrm{NMR}\left(90 \mathrm{MHz}, \mathrm{CDCl}_{3}\right) \delta 170.7,139.6,125.3,73.0,72.2$, 67.1, 63.0, 44.5, 38.9, 36.3, 26.2, 26.1, 18.5, 18.3, 17.9, -4.21, -4.50, -5.10, -5.12. $[\alpha]^{20}{ }_{\mathrm{D}}$ -6.4 (c 0.90, $\left.\mathrm{CH}_{2} \mathrm{Cl}_{2}\right) \cdot[\alpha]^{20}{ }_{\mathrm{D}}+14\left(\mathrm{c} 0.20, \mathrm{CH}_{2} \mathrm{Cl}_{2}\right)$. IR $\left(\mathrm{CH}_{2} \mathrm{Cl}_{2}\right): 1728,1424 \mathrm{~cm}^{-1}$. HRMS (ESI) calcd for $\mathrm{C}_{23} \mathrm{H}_{47} \mathrm{O}_{5} \mathrm{Si}_{2}(\mathrm{M}+\mathrm{H})^{+}: 459.2962$, found: 459.2960 .

\section{2-Allyl-6-[4,5-bis-(tert-butyl-dimethyl-silanyloxy)-2-methyl-pent-1-enyl]-4-}

triethylsilanyloxy-tetrahydro-pyran (13) To a flame-dried flask under Ar, an allylMgBr solution $\left(1.0 \mathrm{M}\right.$ in $\left.\mathrm{Et}_{2} \mathrm{O}, 0.37 \mathrm{~mL}, 0.37 \mathrm{mmol}\right)$ was added dropwise into a solution of the lactone $(57 \mathrm{mg}, 0.12 \mathrm{mmol})$ in $\mathrm{Et}_{2} \mathrm{O}(1.2 \mathrm{~mL})$ at $-78^{\circ} \mathrm{C}$. The suspension was stirred for $0.5 \mathrm{~h}$, before quenched with $\mathrm{H}_{2} \mathrm{O}(1.2 \mathrm{~mL})$ and then diluted with EtOAc (25 mL). The mixture was dried over $\mathrm{Na}_{2} \mathrm{SO}_{4}$, and the solvents were removed under reduced pressure to afford the hemi-ketal $\left(\mathrm{R}_{\mathrm{f}}=0.60,1: 1\right.$ EtOAc:hexanes $)$ as colorless oil $(53 \mathrm{mg})$. This material was used without further purification. To a solution of the hemiketal $(53 \mathrm{mg}, 0.11 \mathrm{mmol})$ in $\mathrm{CH}_{2} \mathrm{Cl}_{2}(1.1 \mathrm{~mL})$ a flame-dried flask under $\mathrm{Ar}$ at $-78^{\circ} \mathrm{C}$, were added $\mathrm{Et}_{3} \mathrm{SiH}(0.18 \mathrm{~mL}, 1.1 \mathrm{mmol})$ in one portion and TFA $(41 \mu \mathrm{L}, 0.55 \mathrm{mmol})$ 
sequentially. The solution was then warmed to $-40^{\circ} \mathrm{C}$ and kept stirring for $0.5 \mathrm{~h}$, before quenched with a saturated $\mathrm{NaHCO}_{3}$ solution $(1.1 \mathrm{~mL})$. The mixture was diluted with $\mathrm{Et}_{2} \mathrm{O}(25 \mathrm{~mL})$ and dried over $\mathrm{Na}_{2} \mathrm{SO}_{4}$. The solvents were then removed under reduced pressure. Flash chromatography (silica, 5\% EtOAc in hexanes) afforded the tetrahydropyran $\left(\mathrm{R}_{\mathrm{f}}=0.50,10 \%\right.$ EtOAc in hexanes) as pale yellow oil $(55 \mathrm{mg}, 76 \%) .{ }^{1} \mathrm{H}$ NMR (360 MHz, CDCl $) \delta 5.84(\mathrm{~m}, 1 \mathrm{H}), 5.20(\mathrm{~d}, \mathrm{~J}=7.9,1 \mathrm{H}), 5.07-4.99(\mathrm{~m}, 2 \mathrm{H}), 4.54$ $(\mathrm{ddd}, \mathrm{J}=13.5,8.3,5.2,1 \mathrm{H}), 4.18(\mathrm{~m}, 1 \mathrm{H}), 3.88(\mathrm{~m}, 1 \mathrm{H}), 3.75(\mathrm{ddd}, \mathrm{J}=11.8,11.8,5.8$, $1 \mathrm{H}), 3.52-3.36(\mathrm{~m}, 2 \mathrm{H}), 2.33-2.25(\mathrm{~m}, 2 \mathrm{H}), 2.13(\mathrm{dd}, \mathrm{J}=13.3,6.8,1 \mathrm{H}), 2.01(\mathrm{dd}, \mathrm{J}=$ 13.3, 6.8, 1H), $1.70(\mathrm{~s}, 3 \mathrm{H}), 1.64-1.19(\mathrm{~m}, 4 \mathrm{H}), 0.96(\mathrm{t}, \mathrm{J}=8.0,9 \mathrm{H}), 0.89(\mathrm{~s}, 9 \mathrm{H}), 0.87$ (s, 9H), 0.58 (q, J = 8.0, 6H), 0.045 (s, 3H), 0.041 (s, 3H), 0.035 (s, 6H). ${ }^{13} \mathrm{C}$ NMR $(90$ $\left.\mathrm{MHz}, \mathrm{CDCl}_{3}\right) \delta 135.5,135.3,129.1,116.5,72.7,71.3,69.3,67.5,65.2,44.9,40.9,39.8$ 38.9, 26.2, 26.1, 18.6, 17.9, 7.1, 5.1, -4.2, -4.5, -5.06, -5.14. $[\alpha]^{20}{ }_{\mathrm{D}}-3.3\left(\mathrm{c} 0.30, \mathrm{CH}_{2} \mathrm{Cl}_{2}\right)$. IR $\left(\mathrm{CH}_{2} \mathrm{Cl}_{2}\right): 1471,1081 \mathrm{~cm}^{-1}$. HRMS (ESI) calcd for $\mathrm{C}_{32} \mathrm{H}_{67} \mathrm{O}_{4} \mathrm{Si}_{3}(\mathrm{M}+\mathrm{H})^{+}:$599.4347, found: 599.4353 .

\section{2-Allyl-6-[3-(2,2-dimethyl-[1,3]dioxolan-4-yl)-2-methyl-propenyl]-tetrahydro-pyran-}

4-ol (14) To a solution of the silyl ether $(180 \mathrm{mg}, 0.30 \mathrm{mmol})$ in THF $(4.8 \mathrm{~mL})$, was added a TBAF solution (1.0 M in THF, $1.2 \mathrm{~mL}, 1.2 \mathrm{mmol})$ and stirred for $6 \mathrm{~h}$. The reaction mixture was then diluted with EtOAc $(20 \mathrm{~mL})$ and brine $(5 \mathrm{~mL})$. The aqueous layer was extracted with EtOAc $(2 \times 10 \mathrm{~mL})$. The combined organics were dried over $\mathrm{Na}_{2} \mathrm{SO}_{4}$ and concentrated under reduced pressure to give the triol $\left(\mathrm{R}_{\mathrm{f}}=0.15\right.$, EtOAc $)$ as pale yellow oil (69 mg). To a flame-dried flask under Ar, the triol was re-dissolved in $\mathrm{CH}_{2} \mathrm{Cl}_{2}(3.0 \mathrm{~mL})$, and dimethoxypropane $(180 \mu \mathrm{L}, 1.5 \mathrm{mmol})$ and $\mathrm{TsOH} \bullet \mathrm{H}_{2} \mathrm{O}(6 \mathrm{mg}$, $0.03 \mathrm{mmol}$ ) were added. The reaction were stirred for $16 \mathrm{~h}$, before quenched with a 
saturated $\mathrm{NaHCO}_{3}$ solution $(3 \mathrm{~mL})$ and then diluted with EtOAc $(30 \mathrm{~mL})$. The organic solution was separated, dried over $\mathrm{Na}_{2} \mathrm{SO}_{4}$, and concentrated under reduced pressure. Flash chromatography (silica, $40 \%$ EtOAc in hexanes) afforded the acetonide $\left(\mathrm{R}_{\mathrm{f}}=0.40\right.$, 1:1 EtOAc: hexanes) as yellow oil (72 mg, 81\%). ${ }^{1} \mathrm{H}$ NMR $\left(360 \mathrm{MHz}, \mathrm{CDCl}_{3}\right) \delta 5.78(\mathrm{~m}$, $1 \mathrm{H}), 5.20(\mathrm{~d}, \mathrm{~J}=8.5,1 \mathrm{H}), 5.06-4.98(\mathrm{~m}, 2 \mathrm{H}), 4.50(\mathrm{~m}, 1 \mathrm{H}), 4.21-4.14(\mathrm{~m}, 2 \mathrm{H}), 3.94$ $(\mathrm{dd}, \mathrm{J}=8.1,6.0,1 \mathrm{H}), 3.84(\mathrm{~m}, 1 \mathrm{H}), 3.49(\mathrm{dd}, \mathrm{J}=7.6,7.6,1 \mathrm{H}), 2.37(\mathrm{dd}, \mathrm{J}=13.7,5.8$, 1H), $2.29(\mathrm{dd}, \mathrm{J}=14.3,7.7,1 \mathrm{H}), 2.20-2.10(\mathrm{~m}, 2 \mathrm{H}), 1.70(\mathrm{~s}, 3 \mathrm{H}), 1.68-1.42(\mathrm{~m}, 4 \mathrm{H})$, $1.37(\mathrm{~s}, 3 \mathrm{H}), 1.31(\mathrm{~s}, 3 \mathrm{H}) .{ }^{13} \mathrm{C} \mathrm{NMR}\left(90 \mathrm{MHz}, \mathrm{CDCl}_{3}\right) \delta$ 135.7, 134.9, 128.4, 117.0, $109.1,74.8,71.2,69.4,69.0,64.9,43.8,40.8,38.8,37.9,27.2,26.0,17.7 .[\alpha]^{20}{ }_{D}+3.6(\mathrm{c}$ 0.22, $\left.\mathrm{CH}_{2} \mathrm{Cl}_{2}\right)$. IR $\left(\mathrm{CH}_{2} \mathrm{Cl}_{2}\right): 1423 \mathrm{~cm}^{-1}$. HRMS (ESI) calcd for $\mathrm{C}_{17} \mathrm{H}_{29} \mathrm{O}_{4}(\mathrm{M}+\mathrm{H})^{+}$: 297.2066, found: 297.2072.

\section{2-Allyl-6-[3-(2,2-dimethyl-[1,3]dioxolan-4-yl)-2-methyl-propenyl]-tetrahydro-pyran-}

4-one (15) To a solution of the alcohol (72 $\mathrm{mg}, 0.24 \mathrm{mmol})$ and $\mathrm{NaOAc}(16 \mathrm{mg}, 0.20$ mmol) in $\mathrm{CH}_{2} \mathrm{Cl}_{2}(2.5 \mathrm{~mL})$, was added PCC (108 mg, $\left.0.50 \mathrm{mmol}\right)$ and stirred for $1.5 \mathrm{~h}$. The reaction mixture was then diluted with $\mathrm{Et}_{2} \mathrm{O}(7.5 \mathrm{~mL})$ and filter through a silica plug, which was washed with $\mathrm{Et}_{2} \mathrm{O}(2.0 \mathrm{~mL})$. The combined organics were concentrated under reduced pressure. Flash chromatography (silica, 20\% EtOAc in hexanes) afforded the ketone $\left(\mathrm{R}_{\mathrm{f}}=0.60,1: 1\right.$ EtOAc: hexanes $)$ as pale yellow oil (53 mg, 74\%). ${ }^{1} \mathrm{H}$ NMR (360 $\left.\mathrm{MHz}, \mathrm{CDCl}_{3}\right) \delta 5.79(\mathrm{~m}, 1 \mathrm{H}), 5.30(\mathrm{dd}, \mathrm{J}=7.6,1.3,1 \mathrm{H}), 5.11-5.06(\mathrm{~m}, 2 \mathrm{H}), 4.30$ (ddd, $\mathrm{J}=13.8,7.9,6.0,1 \mathrm{H}), 4.20(\mathrm{ddd}, \mathrm{J}=12.9,12.9,6.5,1 \mathrm{H}), 3.97(\mathrm{dd}, \mathrm{J}=8.0,6.0,1 \mathrm{H}), 3.67$ (m, 1H), $3.51(\mathrm{dd}, \mathrm{J}=7.9,7.1,1 \mathrm{H}), 2.47-2.14(\mathrm{~m}, 8 \mathrm{H}), 1.69(\mathrm{~s}, 3 \mathrm{H}), 1.38(\mathrm{~s}, 3 \mathrm{H}), 1.32$ (s, 3H). ${ }^{13} \mathrm{C}$ NMR $\left(90 \mathrm{MHz}, \mathrm{CDCl}_{3}\right) \delta 206.9,136.8,133.5,126.8,118.2,109.2,76.5$,

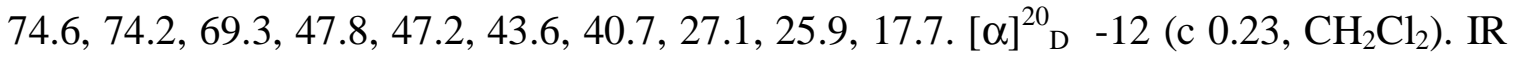


$\left(\mathrm{CH}_{2} \mathrm{Cl}_{2}\right): 1715,1064 \mathrm{~cm}^{-1}$. HRMS (ESI) calcd for $\mathrm{C}_{17} \mathrm{H}_{27} \mathrm{O}_{4}(\mathrm{M}+\mathrm{H})^{+}: 295.1909$, found: 295.1915.

\section{2-Allyl-6-[3-(2,2-dimethyl-[1,3]dioxolan-4-yl)-2-methyl-propenyl]-4-methylene-}

tetrahydropyran (16) To a flame-dried flask under $\mathrm{Ar},{ }^{\mathrm{n}} \mathrm{BuLi}$ (1.5 M solution in hexanes, $0.24 \mathrm{~mL})$ was added dropwise into a solution of $\mathrm{MePh}_{3} \mathrm{PBr}(129 \mathrm{mg}, 0.36 \mathrm{mmol})$ in anhydrous THF $(2.1 \mathrm{~mL})$ at $-78^{\circ} \mathrm{C}$. The reaction mixture was stirred for $15 \mathrm{~min}$, before warmed to $0^{\circ} \mathrm{C}$ and continued to stir for $20 \mathrm{~min}$. A solution of the ketone $(53 \mathrm{mg}$, $0.18 \mathrm{mmol})$ in THF $(2.1 \mathrm{~mL})$ was then added and stirred at $\mathrm{rt}$ for $1.5 \mathrm{~h}$. The reaction was quenched with $\mathrm{H}_{2} \mathrm{O}(4 \mathrm{~mL})$ and the aqueous layer was extracted with ether ( $\left.3 \mathrm{X} 4 \mathrm{~mL}\right)$. The combined organic solution was dried over $\mathrm{Na}_{2} \mathrm{SO}_{4}$ and concentrated under reduced pressure. Flash chromatography (silica, 5\% EtOAc in hexanes) afforded the methylene tetrahydopyran $\left(\mathrm{R}_{\mathrm{f}}=0.45,20 \%\right.$ EtOAc in hexanes) as slightly yellow oil (41 mg, 78\%). ${ }^{1} \mathrm{H}$ NMR $\left(500 \mathrm{MHz}, \mathrm{CDCl}_{3}\right) \delta 5.83(\mathrm{~m}, 1 \mathrm{H}), 5.30(\mathrm{dd}, \mathrm{J}=7.5,1.0,1 \mathrm{H}), 5.06(\mathrm{~m}, 2 \mathrm{H})$, $4.73(\mathrm{dd}, \mathrm{J}=1.5,1.5,2 \mathrm{H}), 4.22(\mathrm{ddd}, \mathrm{J}=13.2,6.1,6.1,1 \mathrm{H}), 4.01(\mathrm{~m}, 1 \mathrm{H}), 4.00(\mathrm{dd}, \mathrm{J}=$ 7.6, 5.7, 1H), $3.54(\mathrm{dd}, \mathrm{J}=7.5,7.5,1 \mathrm{H}), 3.36(\mathrm{~m}, 1 \mathrm{H}), 2.41(\mathrm{dd}, \mathrm{J}=14.0,6.3,2 \mathrm{H}), 2.27-$ $2.12(\mathrm{~m}, 2 \mathrm{H}), 2.03(\mathrm{ddd}, \mathrm{J}=11.5,11.5,1.2,1 \mathrm{H}), 1.92(\mathrm{ddd}, \mathrm{J}=11.5,11.5,1.2,1 \mathrm{H}), 1.72$ $(\mathrm{d}, \mathrm{J}=1.0,3 \mathrm{H}), 1.41(\mathrm{~s}, 3 \mathrm{H}), 1.35(\mathrm{~s}, 3 \mathrm{H}) .{ }^{13} \mathrm{C} \mathrm{NMR}\left(90 \mathrm{MHz}, \mathrm{CDCl}_{3}\right) \delta$ 144.6, 135.7, $134.8,128.4,117.1,109.1,108.9,78.0,75.7,74.8,69.5,43.8,41.0,40.2,27.2,26.0,17.7$. HRMS (EI) calcd for $\mathrm{C}_{18} \mathrm{H}_{28} \mathrm{O}_{3}\left(\mathrm{M}^{+}\right), 292.2038$, found 292.2031.

7-Hydroxy-5-methyl-hepta-2,4-dienoic acid ethyl ester (18) To a flame-dried flask under Ar, a LiHMDS solution (1.0 M in THF, $34.5 \mathrm{~mL}, 34.5 \mathrm{mmol}$ ) was added dropwise into a solution of triethyl phosphonoacetate $(6.91 \mathrm{~mL}, 34.5 \mathrm{mmol})$ in THF (34 mL) and stirred for $15 \mathrm{~min}$. at $0^{\circ} \mathrm{C}$. A solution of the enal $(3.95 \mathrm{~g}, 17.2 \mathrm{mmol})$ in $\mathrm{THF}(52 \mathrm{~mL})$ was 
then added. The reaction mixture was warmed to rt and stirred for $1 \mathrm{~h}$, before diluted with $\mathrm{Et}_{2} \mathrm{O}(100 \mathrm{~mL})$ and quenched with $\mathrm{H}_{2} \mathrm{O}(30 \mathrm{~mL})$. The aqueous solution was extracted with $\mathrm{Et}_{2} \mathrm{O}(30 \mathrm{~mL})$, and the combined organics were dried over $\mathrm{Na}_{2} \mathrm{SO}_{4}$ and concentrated under reduced pressure. Flash chromatography (silica, 5\% EtOAc in hexanes) afforded the dienoate $\left(\mathrm{R}_{\mathrm{f}}=0.40,10 \%\right.$ EtOAc in hexanes $)$ as colorless oil $(5.03 \mathrm{~g}, 98 \%) .{ }^{1} \mathrm{H}$ NMR $\left(360 \mathrm{MHz}, \mathrm{CDCl}_{3}\right) \delta 7.54(\mathrm{dd}, \mathrm{J}=15.3,11.8,1 \mathrm{H}), 6.05(\mathrm{~d}, \mathrm{~J}=11.6,1 \mathrm{H}), 5.75(\mathrm{~d}, \mathrm{~J}=$ $15.1,1 \mathrm{H}), 4.19(\mathrm{~g}, \mathrm{~J}=7.5,2 \mathrm{H}), 3.70(\mathrm{t}, \mathrm{J}=6.5,2 \mathrm{H}), 2.50(\mathrm{t}, \mathrm{J}=6.6,2 \mathrm{H}), 1.90(\mathrm{~s}, 3 \mathrm{H})$, $1.29(\mathrm{t}, \mathrm{J}=7.4,3 \mathrm{H}), 0.87(\mathrm{~s}, 9 \mathrm{H}), 0.04(\mathrm{~s}, 6 \mathrm{H}) .{ }^{13} \mathrm{C} \mathrm{NMR}\left(90 \mathrm{MHz}, \mathrm{CDCl}_{3}\right) \delta 167.7$, $147.1,141.1,125.7,119.5,61.9,60.2,39.2,36.7,26.2,25.2,14.5,-5.1 . \mathrm{IR}\left(\mathrm{CH}_{2} \mathrm{Cl}_{2}\right)$ : 1704, 1637, 1156, $1101 \mathrm{~cm}^{-1}$. HRMS (ESI) calcd for $\mathrm{C}_{16} \mathrm{H}_{31} \mathrm{O}_{3} \mathrm{Si}(\mathrm{M}+\mathrm{H})^{+}: 299.2042$, found: 299.2047.

To a solution of the silyl ether $(298 \mathrm{mg}, 1.0 \mathrm{mmol})$ in THF $(10 \mathrm{~mL})$, was added a Py•HF solution $(65 \%$ in Py, $0.3 \mathrm{~mL}, \sim 10.0 \mathrm{mmol})$ and stirred for $4 \mathrm{~h}$. The reaction mixture was diluted with $\mathrm{Et}_{2} \mathrm{O}(30 \mathrm{~mL})$, and quenched with a saturated $\mathrm{NaHCO}_{3}$ solution $(10 \mathrm{~mL})$ along with solid $\mathrm{NaHCO}_{3}$ until no gas was evolved. The aqueous layer was extracted with EtOAc $(10 \mathrm{~mL})$, and the combined organics were dried over $\mathrm{Na}_{2} \mathrm{SO}_{4}$. Evaporation of solvents afforded the alcohol $\left(\mathrm{R}_{\mathrm{f}}=0.30,1: 1\right.$ EtOAc: hexanes $)$ as pale yellow oil (169 mg, 92\%) without further purification. ${ }^{1} \mathrm{H}$ NMR $\left(360 \mathrm{MHz}, \mathrm{CDCl}_{3}\right) \delta$ $7.54(\mathrm{dd}, \mathrm{J}=15.1,11.7,1 \mathrm{H}), 6.09(\mathrm{~d}, \mathrm{~J}=11.7,1 \mathrm{H}), 5.77(\mathrm{~d}, \mathrm{~J}=15.5,1 \mathrm{H}), 4.17(\mathrm{q}, \mathrm{J}=$ $7.2,2 \mathrm{H}), 3.72(\mathrm{t}, \mathrm{J}=6.7,2 \mathrm{H}), 2.55(\mathrm{t}, \mathrm{J}=6.7,2 \mathrm{H}), 1.90(\mathrm{~s}, 3 \mathrm{H}), 1.27(\mathrm{t}, \mathrm{J}=7.2,3 \mathrm{H}) .{ }^{13} \mathrm{C}$ NMR (90 MHz, $\left.\mathrm{CDCl}_{3}\right) \delta 167.9,146.4,140.5,126.1,119.7,60.9,60.4,36.2,24.7,14.5$. IR $\left(\mathrm{CH}_{2} \mathrm{Cl}_{2}\right): 1704,1637,1154 \mathrm{~cm}^{-1}$. HRMS (ESI) calcd for $\mathrm{C}_{10} \mathrm{H}_{17} \mathrm{O}_{3}(\mathrm{M}+\mathrm{H})^{+}:$: 185.1178 , found: 185.1168 . 
7-Hydroxy-5-methyl-nona-2,4,8-trienoic acid ethyl ester (19) A solution of DessMartin periodinane (15\% in $\mathrm{CH}_{2} \mathrm{Cl}_{2}, 17 \mathrm{~mL}, 8.14 \mathrm{mmol}$ ) was added into the alcohol (1.00 $\mathrm{g}, 5.43 \mathrm{mmol}$ ) at $0^{\circ} \mathrm{C}$. The reaction mixture was warmed to $\mathrm{rt}$ and kept stirring for $1 \mathrm{~h}$, before quenched with isopropanol $(6 \mathrm{~mL})$ and stirred for 5 more minutes. The mixture was filtered through silica, which was then eluted with EtOAc. The combined organics were concentrated under reduced pressure. Flash chromatography (silica, 30\% EtOAc in hexanes) afforded the aldehyde $\left(\mathrm{R}_{\mathrm{f}}=0.35,30 \%\right.$ EtOAc in hexanes $)$ as pale yellow oil (602 mg, 61\%). ${ }^{1} \mathrm{H}$ NMR (360 MHz, $\left.\mathrm{CDCl}_{3}\right) \delta 9.59(\mathrm{t}, \mathrm{J}=1.8,1 \mathrm{H}), 7.39(\mathrm{dd}, \mathrm{J}=15.1$, $11.7,1 \mathrm{H}), 6.21(\mathrm{~d}, \mathrm{~J}=11.7,1 \mathrm{H}), 5.83(\mathrm{~d}, \mathrm{~J}=15.1,1 \mathrm{H}), 4.16(\mathrm{q}, \mathrm{J}=7.2,2 \mathrm{H}), 3.40(\mathrm{~s}, 2 \mathrm{H})$, $1.89(\mathrm{~s}, 3 \mathrm{H}), 1.25(\mathrm{t}, \mathrm{J}=7.2,3 \mathrm{H}) .{ }^{13} \mathrm{C} \mathrm{NMR}\left(90 \mathrm{MHz}, \mathrm{CDCl}_{3}\right) \delta$ 197.5, 167.2, 139.2, 139.0, 127.8, 121.4, 60.5, 47.9, 25.3, 14.4. IR $\left(\mathrm{CH}_{2} \mathrm{Cl}_{2}\right): 1718,1706,1638,1158 \mathrm{~cm}^{-1}$. HRMS (ESI) calcd for $\mathrm{C}_{10} \mathrm{H}_{15} \mathrm{O}_{3}(\mathrm{M}+\mathrm{H})^{+}:$183.1021, found: 183.1036 .

To a flame-dried flask under Ar, a vinylMgBr solution (1.0 M in THF, $0.93 \mathrm{~mL}$, $0.93 \mathrm{mmol}$ ) was added dropwise into a solution of the aldehyde $(67 \mathrm{mg}, 0.37 \mathrm{mmol})$ in THF $(2.8 \mathrm{~mL})$ at $-78^{\circ} \mathrm{C}$. The reaction mixture was stirred for $2 \mathrm{~h}$, before quenched with a saturated $\mathrm{NH}_{4} \mathrm{Cl}$ solution $(0.9 \mathrm{~mL})$, warmed to $\mathrm{rt}$, and diluted with EtOAc $(20 \mathrm{~mL})$. The organic solution was dried over $\mathrm{Na}_{2} \mathrm{SO}_{4}$ and concentrated under reduced pressure. Flash chromatography (silica, 30\% EtOAc in hexanes) afforded the allylic alcohol $\left(\mathrm{R}_{\mathrm{f}}=0.50\right.$, 1:1 EtOAc: hexanes) as brown oil (38 mg, 49\%). ${ }^{1} \mathrm{H}$ NMR (360 MHz, $\left.\mathrm{CDCl}_{3}\right) \delta 7.53$ (dd, $\mathrm{J}=15.1,11.7,1 \mathrm{H}), 6.11(\mathrm{~d}, \mathrm{~J}=11.7,1 \mathrm{H}), 5.88(\mathrm{~m}, 1 \mathrm{H}), 5.77(\mathrm{~d}, \mathrm{~J}=15.1,1 \mathrm{H}), 5.25(\mathrm{~d}, \mathrm{~J}$ $=17.2,1 \mathrm{H}), 5.11(\mathrm{~d}, \mathrm{~J}=10.4,1 \mathrm{H}), 4.17(\mathrm{~g}, \mathrm{~J}=7.1,2 \mathrm{H}), 2.62(\mathrm{dd}, \mathrm{J}=13.4,8.2,1 \mathrm{H}), 2.41$ $(\mathrm{dd}, \mathrm{J}=13.4,5.4,1 \mathrm{H}), 1.91(\mathrm{~s}, 3 \mathrm{H}), 1.27(\mathrm{t}, \mathrm{J}=7.1,3 \mathrm{H}) .{ }^{13} \mathrm{C} \mathrm{NMR}\left(90 \mathrm{MHz}, \mathrm{CDCl}_{3}\right) \delta$ $167.7,145.7,140.5,140.3,126.7,120.0,115.3,71.6,60.3,40.6,25.3,14.5$. IR $\left(\mathrm{CH}_{2} \mathrm{Cl}_{2}\right)$ : 
1707, 1640, 1632, $1157 \mathrm{~cm}^{-1}$. HRMS (ESI) calcd for $\mathrm{C}_{12} \mathrm{H}_{19} \mathrm{O}_{3}(\mathrm{M}+\mathrm{H})^{+}: 211.1334$, found:211.1334.

7-(tert-Butyl-dimethyl-silanyloxy)-5-methyl-nona-2,4,8-trienoic acid (4) In a flame-dried flask under Ar, a solution of the allylic alcohol (195 mg, $0.93 \mathrm{mmol})$, TBSCl (280 mg, $1.86 \mathrm{mmol})$ and $\mathrm{Im}(190 \mathrm{mg}, 2.79 \mathrm{mmol})$ in DMF (2.0 mL) was kept stirring for $20 \mathrm{~h}$, before diluted with $\mathrm{Et}_{2} \mathrm{O}(40 \mathrm{~mL})$ and quenched with $\mathrm{H}_{2} \mathrm{O}(10 \mathrm{~mL})$. The organic solution was dried over $\mathrm{Na}_{2} \mathrm{SO}_{4}$ and concentrated under reduced pressure. Flash chromatography (silica, $10 \%$ EtOAc in hexanes) afforded the TBS ether $\left(\mathrm{R}_{\mathrm{f}}=0.32,10 \%\right.$ EtOAc in hexanes) as pale yellow oil (254 mg, 85\%). ${ }^{1} \mathrm{H}$ NMR $\left(360 \mathrm{MHz}, \mathrm{CDCl}_{3}\right) \delta 7.53$ $(\mathrm{dd}, \mathrm{J}=15.1,11.6,1 \mathrm{H}), 6.05(\mathrm{~d}, \mathrm{~J}=11.6,1 \mathrm{H}), 5.83(\mathrm{dd}, \mathrm{J}=10.4,6.1,1 \mathrm{H}), 5.78(\mathrm{dd}, \mathrm{J}=$ $10.4,6.6,1 \mathrm{H}), 5.75(\mathrm{~d}, \mathrm{~J}=15.3,1 \mathrm{H}), 5.17(\mathrm{ddd}, \mathrm{J}=17.1,1.4,1.4,1 \mathrm{H}), 5.04(\mathrm{ddd}, \mathrm{J}=$ $10.3,1.3,1.3,1 \mathrm{H}), 4.22(\mathrm{~m}, 1 \mathrm{H}), 4.19(\mathrm{q}, \mathrm{J}=7.1,2 \mathrm{H}), 2.58(\mathrm{dd}, \mathrm{J}=13.2,7.7,1 \mathrm{H}), 2.34$ $(\mathrm{dd}, \mathrm{J}=13.2,5.4,1 \mathrm{H}), 1.90(\mathrm{~s}, 3 \mathrm{H}), 1.28(\mathrm{t}, \mathrm{J}=7.1,3 \mathrm{H}), 0.86(\mathrm{~s}, 9 \mathrm{H}), 0.01(\mathrm{~s}, 6 \mathrm{H}) .{ }^{13} \mathrm{C}$ NMR $\left(90 \mathrm{MHz}, \mathrm{CDCl}_{3}\right) \delta 167.6,146.2,141.1,141.0,126.1,119.3,114.1,73.0,60.1$, 41.7, 25.9, 25.7, 18.3, 14.4, -2.9, -4.4. IR $\left(\mathrm{CH}_{2} \mathrm{Cl}_{2}\right): 1708,1631 \mathrm{~cm}^{-1}$. HRMS (ESI) calcd for $\mathrm{C}_{18} \mathrm{H}_{33} \mathrm{O}_{3} \mathrm{Si}(\mathrm{M}+\mathrm{H})^{+}: 325.2199$, found: 325.2205.

To a solution of the ester $(241 \mathrm{mg}, 0.74 \mathrm{mmol})$ in $\mathrm{EtOH}(3.7 \mathrm{~mL})$, was added dropwise an aqueous $\mathrm{NaOH}$ solution $(1.0 \mathrm{M}, 1.85 \mathrm{~mL})$ at $0^{\circ} \mathrm{C}$. The reaction mixture was then warmed to rt and stirred for $20 \mathrm{~h}$ before an aqueous $\mathrm{HCl}$ solution was added (1.0 M, $1.85 \mathrm{~mL})$. EtOAc $(20 \mathrm{~mL})$ was added and allowed to partition. The aqueous layer was extracted with EOAc ( $2 \times 5 \mathrm{~mL}$ ) and the organics were combined, dried over $\mathrm{Na}_{2} \mathrm{SO}_{4}$, and solvents were removed under reduced pressure. The residue was re-dissolved in toluene $(4 \mathrm{~mL})$ and then rotavapped to afford the acid $\left(\mathrm{R}_{\mathrm{f}}=0.56\right.$, EtOAc) as pale yellow 
viscous oil (208 $\mathrm{mg}, 95 \%)$. This material was used without further purification. This is a known compound. \{Hoye, T. R.; Hu, M. J.Am. Chem. Soc. 2003, 125, 9576.\}

5-(6-Allyl-4-methylene-tetrahydro-pyran-2-yl)-1-(tert-butyl-dimethyl-silanyloxy)-4methyl-pent-4-en-2-ol (3) To a solution of the acetonide (29 mg, $0.1 \mathrm{mmol})$ in EtOH $(0.33 \mathrm{~mL})$ and $\mathrm{CH}_{2} \mathrm{Cl}_{2}(0.33 \mathrm{~mL})$ at $0^{\circ} \mathrm{C}$, was added TFA $(0.33 \mathrm{~mL})$ dropwise and stirred for $10 \mathrm{~min}$. The solvents were removed under reduced pressure, and the residue was coevaporated with PhMe ( 2 X $2 \mathrm{~mL}$ ) to remove TFA to afford the diol. To a flame-dried flask under $\mathrm{Ar}, \mathrm{Et}_{3} \mathrm{~N}(56 \mu \mathrm{L}, 0.4 \mathrm{mmol})$ was added dropwise into a solution of the diol, TBSCl $(38 \mathrm{mg}, 0.25 \mathrm{mmol})$, DMAP $(2 \mathrm{mg}, 16 \mu \mathrm{mol})$ in $\mathrm{CH}_{2} \mathrm{Cl}_{2}(1.0 \mathrm{~mL})$ at $0^{\circ} \mathrm{C}$. The reaction mixture was warmed to $\mathrm{rt}$ and stirred for $1.5 \mathrm{~h}$, before quenched with $\mathrm{H}_{2} \mathrm{O}(0.5$ $\mathrm{mL})$. The aqueous layer was extracted with $\mathrm{Et}_{2} \mathrm{O}(2 \times 5 \mathrm{~mL})$, and the combined organic solution was dried over $\mathrm{Na}_{2} \mathrm{SO}_{4}$ and concentrated under reduced pressure. Flash chromatography (silica, 20\% EtOAc in hexanes) afforded the alcohol $\left(\mathrm{R}_{\mathrm{f}}=0.35,20 \%\right.$ EtOAc in hexanes) as colorless oil $(35 \mathrm{mg}, 97 \%) .{ }^{1} \mathrm{H}$ NMR $\left(360 \mathrm{MHz}, \mathrm{CDCl}_{3}\right) \delta 5.83(\mathrm{~m}$, $1 \mathrm{H}), 5.30(\mathrm{~d}, \mathrm{~J}=7.8,1 \mathrm{H}), 5.10-5.03(\mathrm{~m}, 2 \mathrm{H}), 4.73(\mathrm{~s}, 2 \mathrm{H}), 4.01(\mathrm{ddd}, \mathrm{J}=10.8,7.8,2.7$, $1 \mathrm{H}), 3.79$ (dddd. $\mathrm{J}=13.5,10.6,6.7,3.8,1 \mathrm{H}), 3.59(\mathrm{dd}, \mathrm{J}=10.0,3.8,1 \mathrm{H}), 3.44(\mathrm{dd}, \mathrm{J}=$ 10.0, 6.6, 1H), $3.36(\mathrm{~m}, 1 \mathrm{H}), 2.43-2.14(\mathrm{~m}, 6 \mathrm{H}), 2.04(\mathrm{dd}, \mathrm{J}=11.3,11.3,1 \mathrm{H}), 1.92(\mathrm{dd}$, $\mathrm{J}=11.3,11.3,1 \mathrm{H}), 1.72(\mathrm{~d}, \mathrm{~J}=1.2,3 \mathrm{H}), 0.90(\mathrm{~s}, 9 \mathrm{H}), 0.06(\mathrm{~s}, 6 \mathrm{H}) .{ }^{13} \mathrm{C} \mathrm{NMR}(90 \mathrm{MHz}$ $\left.\mathrm{CDCl}_{3}\right) \delta 144.6,136.0,134.7,128.6,117.3,108.8,77.9,75.7,70.1,66.7,43.4,40.8,40.1$, $26.1,18.4,17.4,-5.2$.

7-(tert-Butyl-dimethyl-silanyloxy)-5-methyl-nona-2,4,8-trienoic acid 4-(6-allyl-4methylene-tetrahydro-pyran-2-yl)-1-(tert-butyl-dimethyl-silanyloxymethyl-3methyl-but-3-enyl ester (20)To a flame-dried flask under Ar, trichlorobenzoyl chloride 
(20 $\mu \mathrm{L}, 0.13 \mathrm{mmol})$ was added dropwise into a solution of the acid $(25 \mathrm{mg}, 0.084 \mathrm{mmol})$ and $\mathrm{Et}_{3} \mathrm{~N}(24 \mu \mathrm{L}, 0.17 \mathrm{mmol})$ in PhMe $(0.8 \mathrm{~mL})$. The reaction mixture was stirred for $1 \mathrm{~h}$, before was added a solution of the alcohol (14 $\mathrm{mg}, 0.038 \mathrm{mmol}$ ) and DMAP (6 mg, 0.046 mmol) in PhMe $(0.4 \mathrm{~mL})$. The resultant suspension was stirred for $1 \mathrm{~h}$, and then a saturated $\mathrm{NaHCO}_{3}$ solution $(1.2 \mathrm{~mL})$ was added followed by $\mathrm{Et}_{2} \mathrm{O}(20 \mathrm{~mL})$. The aqueous layer was extracted with $\mathrm{Et}_{2} \mathrm{O}(5 \mathrm{~mL})$, and the combined organic solution was dried over $\mathrm{Na}_{2} \mathrm{SO}_{4}$ and concentrated under reduced pressure. Flash chromatography (silica, 5\% EtOAc in hexanes) afforded the ester $\left(\mathrm{R}_{\mathrm{f}}=0.50,0.55,20 \%\right.$ EtOAc in hexanes $)$ as yellow oil (28 mg, yield:100\%, dr: 3:1). Major diastereomer: ${ }^{1} \mathrm{H}$ NMR $\left(360 \mathrm{MHz}, \mathrm{CDCl}_{3}\right) \delta 7.54$ $(\mathrm{dd}, \mathrm{J}=15.7,11.7,1 \mathrm{H}), 6.06(\mathrm{~d}, \mathrm{~J}=11.7,1 \mathrm{H}), 5.88-5.75(\mathrm{~m}, 2 \mathrm{H}), 5.76(\mathrm{~d}, \mathrm{~J}=15.5,1 \mathrm{H})$, $5.30(\mathrm{~d}, \mathrm{~J}=7.9,1 \mathrm{H}), 5.17(\mathrm{~d}, \mathrm{~J}=17.1,1 \mathrm{H}), 5.10-5.01(\mathrm{~m}, 4 \mathrm{H}), 4.72(\mathrm{~s}, 2 \mathrm{H}), 4.24(\mathrm{dd}, \mathrm{J}$ $=12.3,5.8,1 \mathrm{H}), 3.98(\mathrm{~m}, 1 \mathrm{H}), 3.66(\mathrm{~m}, 2 \mathrm{H}), 3.34(\mathrm{~m}, 1 \mathrm{H}), 2.36-1.83(\mathrm{~m}, 10 \mathrm{H}), 1.90(\mathrm{~s}$, 3H), 1.73 (s, 3H), 0.88 (s, 9H), 0.86 (s, 9H), 0.04 (s, 3H), 0.03 (s, 3H), $0.00(\mathrm{~s}, 6 \mathrm{H}) .{ }^{13} \mathrm{C}$ NMR $\left(90 \mathrm{MHz}, \mathrm{CDCl}_{3}\right.$ ) The carbon spectrum is too complicated to interpret properly. $[\alpha]^{20}{ }_{D}+28\left(\right.$ c $\left.0.05, \mathrm{CH}_{2} \mathrm{Cl}_{2}\right)$. HRMS (ESI) calcd for $\mathrm{C}_{37} \mathrm{H}_{65} \mathrm{O}_{5} \mathrm{Si}_{2}(\mathrm{M}+\mathrm{H})^{+}:$645.4371, found: 645.4370 .

\section{3-Hydroxy-5-hydroxymethyl-3,11-dimethyl-19-methylene-6,21-dioxa-}

bicyclo[15.3.1]heneic osa-2,8,10,14-tetraen-7-one (21) To a solution of the bis-TBS ether $(6.4 \mathrm{mg}, 10 \mu \mathrm{mol})$ in $\mathrm{MeOH}(200 \mu \mathrm{L})$ and $\mathrm{CH}_{2} \mathrm{Cl}_{2}(50 \mu \mathrm{L})$, was added an aqueous $\mathrm{HCl}$ solution $(1.0 \mathrm{M}, 20 \mu \mathrm{L})$ and stirred for $4.5 \mathrm{~h}$. A saturated $\mathrm{NaHCO}_{3}$ solution $(0.1 \mathrm{~mL})$ was then added, and the aqueous solution was extracted with EtOAc $(2 \times 3 \mathrm{~mL})$. The organics were combined, dried over $\mathrm{Na}_{2} \mathrm{SO}_{4}$, and solvents were removed under reduced pressure. Flash chromatography (silica, 1:1 EtOAc: hexanes) afforded the diol $\left(\mathrm{R}_{\mathrm{f}}=0.31\right.$, 
1:1 EtOAc: hexanes) as a slightly yellow solid (3.3 mg, yield: $80 \%$, dr: 3:1).major diastereomer: ${ }^{1} \mathrm{H}$ NMR $\left(360 \mathrm{MHz}, \mathrm{CDCl}_{3}\right) \delta 7.59(\mathrm{~m}, 1 \mathrm{H}), 6.14(\mathrm{~d}, \mathrm{~J}=11.7,1 \mathrm{H}), 5.94-$ $5.76(\mathrm{~m}, 3 \mathrm{H}), 5.32(\mathrm{~d}, \mathrm{~J}=8.0,1 \mathrm{H}), 5.27(\mathrm{ddd}, \mathrm{J}=17.1,1.2,1.2,1 \mathrm{H}), 5.16-5.02(\mathrm{~m}, 4 \mathrm{H})$, $4.72(\mathrm{~s}, 2 \mathrm{H}), 4.29(\mathrm{~m}, 1 \mathrm{H}), 3.98(\mathrm{~m}, 1 \mathrm{H}), 3.74(\mathrm{dd}, \mathrm{J}=12.1,3.1,1 \mathrm{H}), 3.64(\mathrm{dd}, \mathrm{J}=12.1$, 6.4, 1H), $3.34(\mathrm{~m}, 1 \mathrm{H}), 2.64(\mathrm{~m}, 1 \mathrm{H}), 2.45-1.87(\mathrm{~m}, 9 \mathrm{H}), 1.94(\mathrm{~s}, 3 \mathrm{H}), 1.74(\mathrm{~s}, 3 \mathrm{H}) .{ }^{13} \mathrm{C}$ NMR (90 MHz, $\left.\mathrm{CDCl}_{3}\right) \delta 167.9,146.4,144.5,141.5,140.3,134.7,134.5,129.5,126.7$, 119.5, 117.1, 115.5, 109.0, 77.9, 75.7, 73.9, 71.6, 64.6, 40.9, 40.8, 40.1, 29.9, 25.4, 17.4. $[\alpha]^{20}{ }_{D}+11\left(\mathrm{c} 0.07, \mathrm{CH}_{2} \mathrm{Cl}_{2}\right)$. HRMS (ESI) calcd for $\mathrm{C}_{25} \mathrm{H}_{37} \mathrm{O}_{5}(\mathrm{M}+\mathrm{H})^{+}: 417.2641$, found: 417.2644.

To a flame-dried flask under Ar, the Grubbs' second generation catalyst $(0.8 \mathrm{mg}$, $1.0 \mu \mathrm{mol})$ was added into a stirring solution of the hexaene $(4.1 \mathrm{mg}, 10 \mu \mathrm{mol})$ in $\mathrm{CH}_{2} \mathrm{Cl}_{2}$ $(10 \mathrm{~mL})$, and kept stirring for $1 \mathrm{~h}$. The solution was concentrated under reduced pressure. Flash chromatography (silica, 3:2 EtOAc: hexanes) afforded the pentaene $\left(\mathrm{R}_{\mathrm{f}}=0.32,3: 2\right.$ EtOAc: hexanes) as a colorless solid (3.6 mg, yield: 93\%, dr: 1:1). ${ }^{1} \mathrm{H}$ NMR (360 MHz, $\left.\mathrm{CDCl}_{3}\right) \delta 7.58(\mathrm{dd}, \mathrm{J}=15.1,11.6) / 7.45(\mathrm{dd}, \mathrm{J}=15.1,11.5,1 \mathrm{H})^{*}, 6.06(\mathrm{~d}, \mathrm{~J}=11.5,1 \mathrm{H})$, $5.81(\mathrm{~d}, \mathrm{~J}=15.1) / 5.76(\mathrm{~d}, \mathrm{~J}=15.1,1 \mathrm{H})^{*}, 5.76-5.56(\mathrm{~m}, 2 \mathrm{H}), 5.29(\mathrm{~d}, \mathrm{~J}=7.0) / 5.23(\mathrm{~d}, \mathrm{~J}$ $=8.0,1 \mathrm{H})^{*}, 5.18(\mathrm{~m}, 1 \mathrm{H}), 4.73(\mathrm{~s}) / 4.71(\mathrm{~s}, 2 \mathrm{H})^{*}, 4.35(\mathrm{dd}, \mathrm{J}=12.0,7.1) / 4.23(\mathrm{dd}, \mathrm{J}=$ 12.2, 5.5, 1H)*, $3.94(\mathrm{ddd}, \mathrm{J}=17.6,8.4,2.7,1 \mathrm{H}), 3.72(\mathrm{~m}, 2 \mathrm{H}), 3.32(\mathrm{ddd}, \mathrm{J}=11.1,11.1$, 2.3, 1H), $2.82(\mathrm{dd}, \mathrm{J}=13.4,8.3,)^{*} / 2.56-1.62(\mathrm{~m}, 10 \mathrm{H}), 1.99(\mathrm{~s}) / 1.88(\mathrm{~s}, 3 \mathrm{H})^{*}, 1.72$ (s)/1.69 (s, 3H)*. *: due to diastereomers. ${ }^{13} \mathrm{C}$ NMR $\left(90 \mathrm{MHz}, \mathrm{CDCl}_{3}\right)$ The carbon spectrum is too complicated to interpret properly. HRMS (ESI) calcd for $\mathrm{C}_{23} \mathrm{H}_{33} \mathrm{O}_{5}$ $(\mathrm{M}+\mathrm{H})^{+}: 389.2328$, found: 389.2306 . 
(-)-Dactylolide (2) To a solution of the diol (3.6 mg, $9.3 \mu \mathrm{mol})$ in $\mathrm{CH}_{2} \mathrm{Cl}_{2}(100 \mu \mathrm{L})$, was added dropwise a Dess-Martin solution $\left(15 \%\right.$ in $\left.\mathrm{CH}_{2} \mathrm{Cl}_{2}, 77 \mu \mathrm{L}, 37.2 \mu \mathrm{mol}\right)$ at $0^{\circ} \mathrm{C}$ and then warmed to rt. The reaction mixture was stirred for $0.5 \mathrm{~h}$ before diluted with $\mathrm{CH}_{2} \mathrm{Cl}_{2}(1.0 \mathrm{~mL})$ and $\mathrm{Et}_{2} \mathrm{O}(2.0 \mathrm{~mL})$. Pyridine $(5 \mu \mathrm{L}, 91 \mu \mathrm{mol})$ was added and the whole solution was filter through a plug of celite. Solvents were removed under reduced pressure. Flash chromatography (silica, 1:1 EtOAc: hexanes) afforded dactylolide $\left(\mathrm{R}_{\mathrm{f}}=\right.$ 0.46, 3:2 EtOAc: hexanes) as a colorless solid (3.2 mg, 90\%). ${ }^{1} \mathrm{H}$ NMR (500 MHz, $\left.\mathrm{CDCl}_{3}\right) \delta 9.67(\mathrm{~s}, 1 \mathrm{H}), 7.63(\mathrm{dd}, \mathrm{J}=15.1,11.6,1 \mathrm{H}), 6.85(\mathrm{ddd}, \mathrm{J}=16.1,8.6,5.9,1 \mathrm{H})$, $6.15(\mathrm{~d}, \mathrm{~J}=11.6,1 \mathrm{H}), 6.00(\mathrm{~d}, \mathrm{~J}=16.2,1 \mathrm{H}), 5.96(\mathrm{~d}, \mathrm{~J}=15.1,1 \mathrm{H}), 5.32(\mathrm{dd}, \mathrm{J}=11.4$ 2.4, 1H), $5.24(\mathrm{~d}, \mathrm{~J}=8.0,1 \mathrm{H}), 4.75(\mathrm{~s}, 2 \mathrm{H}), 3.97(\mathrm{ddd}, \mathrm{J}=11.1,8.1,2.5,1 \mathrm{H}), 3.95(\mathrm{~d}, \mathrm{~J}=$ 14.3, 1H), 3.32 (dddd, $\mathrm{J}=11.4,9.1,2.5,2.5,1 \mathrm{H}), 3.23(\mathrm{~d}, \mathrm{~J}=14.3,1 \mathrm{H}), 2.54(\mathrm{~d}, \mathrm{~J}=14.0$, 1H), 2.39-2.27 (m, 3H), 2.17 (ddd, $\mathrm{J}=13.2,1.6,1.6,1 \mathrm{H}), 2.11(\mathrm{ddd}, \mathrm{J}=13.0,1.6,1.6$, 1H), 1.96 (m, 2H), 1.86 (s, 3H), $\left.1.72(\mathrm{~s}, 3 \mathrm{H}) .{ }^{13} \mathrm{C} \mathrm{NMR} \mathrm{(90} \mathrm{MHz,} \mathrm{CDCl}_{3}\right) \delta$ 199.3, 197.6, 166.5, 146.2, 144.2, 140.6, 131.7, 131.1, 130.7, 125.8, 120.0, 109.5, 76.7, 75.9, 75.5, 45.1, 41.0, 40.6, 39.94, 39.90, 24.3, 16.3. $[\alpha]^{20}{ }_{\mathrm{D}}=-136(\mathrm{c} 1.2, \mathrm{MeOH}) . \mathrm{IR}\left(\mathrm{CH}_{2} \mathrm{Cl}_{2}\right): 1722$, 1703, 1668, 1638, $1283 \mathrm{~cm}^{-1}$. HRMS (ESI) calcd for $\mathrm{C}_{23} \mathrm{H}_{29} \mathrm{O}_{5}(\mathrm{M}+\mathrm{H})^{+}: 385.2015$, found: 385.2023. 